\title{
Mamíferos de la Reserva Rogitama Biodiversidad (Arcabuco, Boyacá): un hogar en rehabilitación en la Cordillera Oriental de Colombia
}

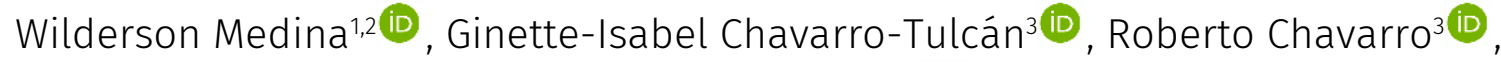 \\ Francisco Sánchez ${ }^{4 *}$ (i)
}

1 Grupo Sistemática Biológica, SisBio. Escuela de Biología, Facultad de Ciencias Básicas, Universidad Pedagógica y Tecnológica de Colombia, Tunja, Colombia.

2 Nicholas School of the Environment, Duke University, Durham, NC 27708, USA.

3 Reserva Natural de la Sociedad Civil Rogitama Biodiversidad, Arcabuco, Colombia.

4 Grupo ECOTONOS, Programa de Biología, Facultad de Ciencias Básicas e Ingeniería, Universidad de Los Llanos, Villavicencio, Colombia.

*Correspondencia: fasbos@gmail.com

\section{Resumen}

En los Andes orientales de Colombia amplias áreas de robledales han sido reemplazadas por cultivos o ganadería. Algunos propietarios privados han decidido convertir sus predios en reservas naturales privadas al ver las consecuencias después de eventos de deforestación. Una de estas áreas es Rogitama Biodiversidad ubicada en el municipio de Arcabuco (Boyacá), la cual se encuentra en proceso de rehabilitación ecológica. Estudiamos la composición de mamíferos como indicador del estado de la reserva después de 40 años de procesos de rehabilitación. Encontramos 22 especies, en su mayoría murciélagos y roedores. Estos resultados reflejan valores de riqueza semejantes a los de otras localidades menos intervenidas de la Cordillera Oriental y sugiere avances en la recuperación de la funcionalidad ecosistémica en Rogitama. Consideramos que Rogitama es un ejemplo de la recuperación vegetal que ha facilitado el regreso de mamíferos silvestres a áreas que han sufrido procesos de defaunación.

Palabras clave: Andes, Inventario biológico, Quercus humboldtii, Reservas Privadas, Restauración ecológica.

\begin{abstract}
In the eastern Andes of Colombia, large areas of oak forests have been replaced by crops or livestock. Some private owners have decided to turn their properties into private nature reserves after seeing the consequences after deforestation events. One of these areas is Rogitama Biodiversity located in the municipality of Arcabuco (Boyacá), which is in the process of ecological rehabilitation. We study the composition of mammals as an indicator of the state of the reserve after 40 years of rehabilitation processes. We found 22 species, mostly bats and rodents. These results reflect values of wealth similar to those of other less intervened localities of the Eastern Cordillera and suggest progress in the recovery of ecosystem functionality in Rogitama. We consider that Rogitama is an example of plant recovery that has Keywords: Biological survey, private natural preserves, Quercus humboldtii, ecological restoration, the Andes
\end{abstract}




\section{INTRODUCCIÓN}

Después de más de 60 años desde la creación de las primeras reservas naturales en Colombia, cerca del 18 \% de la región Andina ha sido delimitada como área protegida. Esto a pesar de la creciente tasa de deforestación que ha llevado a que menos del 30 \% de la cobertura nativa de bosques andinos esté conservada (Armenteras et al. 2013; SánchezCuervo \& Aide 2013). El ecosistema de robledal, un bosque andino dominado por Quercus humboldtii Bonpl. (Fagaceae, Fagales), ha sufrido los efectos de la deforestación a través de procesos como quemas, ganadería, apertura de vías, asentamientos humanos, extracción de productos del bosque y producción de carbón vegetal (Devia \& Arenas 2000; Otálora-Ardila 2003). El robledal de la Cordillera Oriental es el de mayor extensión en el país y la tasa de deforestación anual en departamentos como Boyacá, Santander y Cundinamarca sobrepasa la recuperación natural, por lo que se requieren de manera urgente planes de conservación de sus áreas naturales y de la biodiversidad que contienen (Avella Muñoz \& Cárdenas Camacho 2010).

Para la conservación de esta y otras áreas de importancia ecológica, se ha sugerido la vinculación activa de actores sociales no estatales, ya que contempla la preservación, la restauración ecológica y el sostenimiento de comunidades locales (Seabrook et al. 2011; Dewi et al. 2013). Los predios rurales privados en los Andes ocupan cuatro veces más área que las tierras pertenecientes al Estado (Ibáñez et al. 2012), representando un alto potencial para la conservación de la biodiversidad andina. Su creación y la restauración ecológica se han propuesto como herramientas para mitigar los efectos de la pérdida de ecosistemas naturales (Dobson et al. 1997). Sin embargo, los actuales planes de restauración en Colombia (Ministerio de Ambiente y Desarrollo Sostenible 2015) requieren de mayor apoyo para ser implementados a una mayor escala.

La restauración de un área alterada puede ser evaluada a través de indicadores como la riqueza de especies y su estado de conservación (Clewel \& Aronson 2013). En particular, los mamíferos pueden usarse como indicadores de restauración de ecosistemas ya que responden a cambios de las coberturas vegetales o efectos de borde (Sánchez et al. 2008; Narváez \& Sánchez 2013; Díaz-Pulido et al. 2015). No obstante, no se ha documentado la composición de mamíferos después de prolongados procesos de reforestación en los Andes colombianos. En este trabajo hacemos un análisis preliminar de la composición de la comunidad de mamíferos de una reserva privada en la Cordillera Oriental después de 40 años de rehabilitación ecológica.

\section{MATERIALES Y MÉTODOS}

\section{Área de estudio}

El corredor andino de roble está en de los departamentos de Boyacá y Santander (AvellaMuñoz \& Cárdenas-Camacho 2010). Algunas reservas naturales de la sociedad civil son parte de este corredor, y una de ellas es la Reserva Rogitama Biodiversidad. Esta Reserva se encuentra en el sector Mordoñal de la vereda Peñas Blancas, entre 2.485 y $2.550 \mathrm{msnm}$, con coordenadas $5.797 \mathrm{~N},-73.458 \mathrm{~W}$. Presenta una temperatura promedio de $13{ }^{\circ} \mathrm{C}$ y tiene un patrón de precipitación bimodal con baja precipitación entre diciembre-enero y junioseptiembre (101 mm/mes) y alta entre abril-mayo y octubre-noviembre $(237 \mathrm{~mm} / \mathrm{mes})$ (IDEAM 2021). Rogitama Biodiversidad comprende 29 ha que solían estar dominadas por pastizales y árboles aislados (Figura 1). Actualmente es un mosaico de áreas en distintos estadios sucesionales producto de procesos de regeneración asistida y natural, 
correspondientes a 7 ha de bosque de roble, 16 ha de potreros en proceso de restauración asistida con especies exóticas y nativas, y 6 ha de potrero para manejo productivo. La información suplementaria 1 presenta la historia de la rehabilitación en Rogitama.

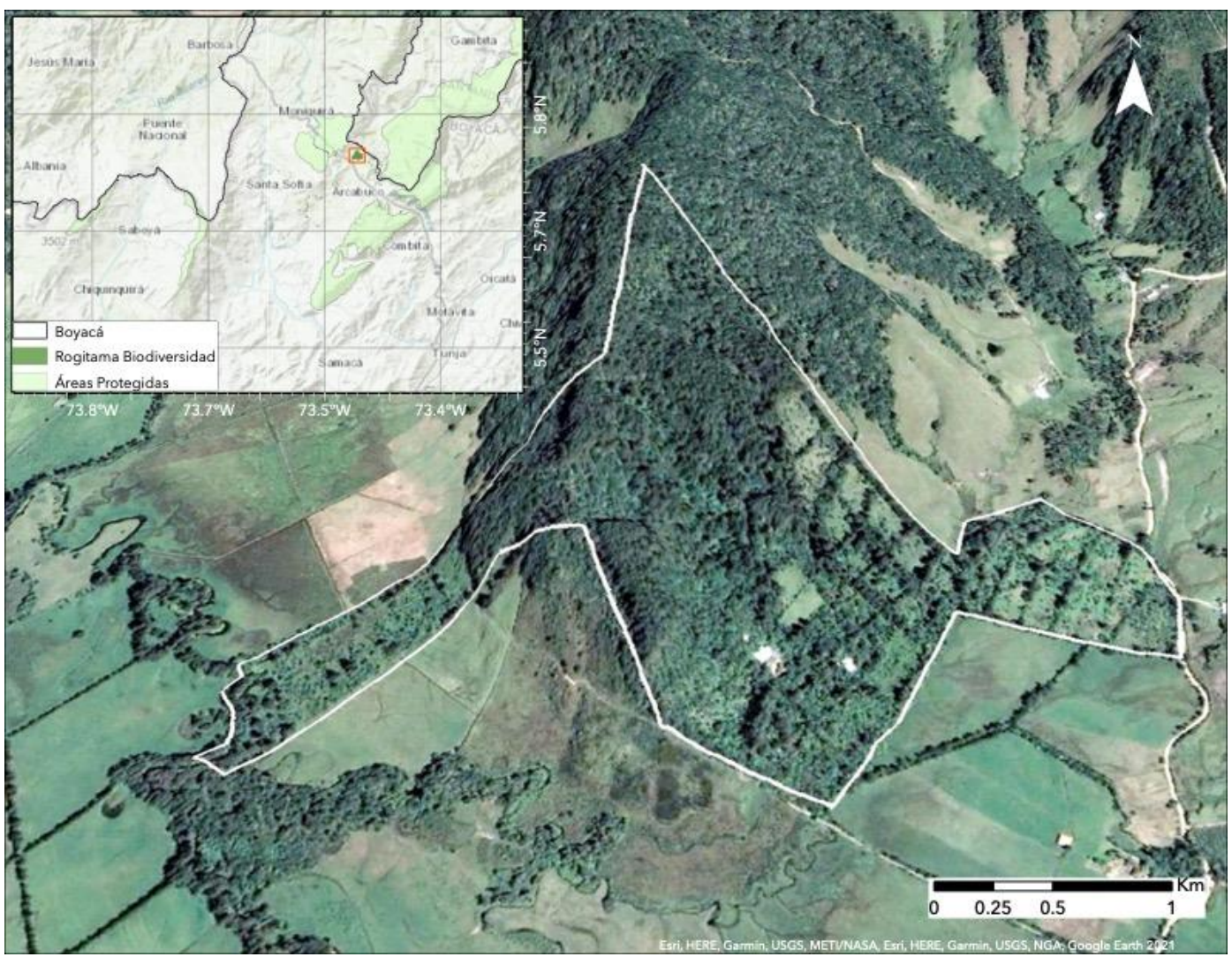

FIGURA 1. Reserva de la sociedad civil Rogitama Biodiversidad; el perimetro blanco en la imagen muestra los límites de la Reserva. La Reserva conserva la biodiversidad local conectándose con otras áreas protegidas mediante extensos corredores biológicos no protegidos. En el recuadro superior está la posición de la Reserva en el Departamento de Boyacá y también se muestran los perímetros de las áreas protegidas del departamento, que se obtuvieron del repositorio de Parques Nacionales en ArcGIS online. Imágenes de ESRI, USGS y Google Earth 2021.

\section{Metodología}

Utilizamos cinco técnicas para muestrear mamíferos entre el 26 y 29 de julio de 2010 y el 13 y 15 de octubre de 2015 en áreas de bosque mixto (áreas con dominancia de robles, baja densidad de pinos y acacias, y algunos elementos de familias nativas), y en bordes de bosque con pastizales. Para capturar murciélagos usamos redes de niebla durante tres noches con un esfuerzo de $864 \mathrm{~h} \cdot \mathrm{m}^{2}$.red. Registramos mamíferos terrestres con trampas Sherman (90 trampas·noche), trampas Tomahawk (6 trampas-noche), y cámaras trampa (Bushnell Trophy Cam; 6 cámaras-noche). Complementamos el inventario con observaciones y capturas incidentales, de algunos visitantes de la Reserva. Seguimos la nomenclatura de Ramírez-Chaves et al. 2021. Los pequeños mamíferos no voladores y murciélagos recolectados fueron depositados en el Museo de Historia Natural de la Universidad Pedagógica y Tecnológica de Colombia, UPTC (números consecutivos de registro WM225-WM233); permiso marco de recolección, resolución 0724 del 4 de julio de 
2014. Seguimos el protocolo de captura y manejo de especímenes de pequeños mamíferos propuesto por Sikes et al. (2016), previa aprobación de la UPTC.

\section{RESULTADOS Y DISCUSIÓN}

Identificamos 22 especies pertenecientes a ocho órdenes y 16 familias y una amplia variedad de gremios tróficos (Tabla 1; Figuras 3-6 e información suplementaria). Los murciélagos y roedores aportaron seis especies. Cinco murciélagos pertenecen a la familia Phyllostomidae y un solo insectívoro aéreo, Myotis cf. oxyotus. También se incluye un individuo de Potos flavus recientemente liberado (Figura 3 en información suplementaria 2). Destacamos el registro a mayor altitud documentado de Didelphis marsupialis (Solari et al. 2013), cohabitando con D. pernigra (Figuras 4a-c en información suplementaria 2). Finalmente, registramos un individuo de Leopardus tigrinus; especie categorizada como vulnerable por la IUCN por la pérdida de hábitat (Payán \& de Oliveira 2016) (Figura 4e en información suplementaria 2).

TABLA 1. Mamíferos de la Reserva Rogitama Biodiversidad en Arcabuco, Boyacá, Colombia.

\begin{tabular}{|c|c|c|c|c|}
\hline ORDEN & Familia & Especie & Método de registro & Gremio Trófico \\
\hline \multirow[t]{2}{*}{ DIDELPHIMORPHIA } & \multirow[t]{2}{*}{ Didelphidae } & Didelphis marsupialis & $\begin{array}{l}\text { Trampa casera tipo } \\
\text { Tomahawk Cámara trampa }\end{array}$ & Omnívoro \\
\hline & & Didelphis pernigra & Cámara trampa & Omnívoro \\
\hline SORICOMORPHA & Soricidae & Cryptotis sp. & $\begin{array}{l}\text { Captura incidental en } \\
\text { Trampa de caída para } \\
\text { invertebrados }\end{array}$ & Insectivoro \\
\hline CINGULATA & Dasypodidae & Dasypus novemcinctus & Encontrado muerto & Insectívoro \\
\hline PILOSA & Megalonychidae & Choloepus hoffmanni** & Observación directa & Folivoro \\
\hline \multirow[t]{4}{*}{ CARNIVORA } & Felidae & Leopardus tigrinus & Cámara trampa & Carnívoro \\
\hline & Canidae & $\begin{array}{l}\text { Urocyon cinereoargenteus } 0 \\
\text { Cerdocyon thous ** }\end{array}$ & Observación directa & Carnívoro \\
\hline & Mustelidae & Neogale frenata & Cámara trampa & Carnívoro \\
\hline & Procyonidae & Potos flavus & Observación directa & Omnívoro \\
\hline \multirow[t]{6}{*}{ RODENTIA } & Sciuridae & Syntheosciurus granatensis & $\begin{array}{l}\text { Cámara trampa; } \\
\text { avistamientos }\end{array}$ & Omnívoro \\
\hline & \multirow[t]{2}{*}{ Cricetidae } & Rhipidomys fulviventer* & Trampa Sherman & Frugivoro \\
\hline & & Rhipidomys sp.* & Trampa Sherman & Frugivoro \\
\hline & Erethizontidae & Coendou rufescens ${ }^{\star \star}$ & Observación directa & Frugivoro \\
\hline & Dasyproctidae & Dasyprocta punctata & Cámara trampa & Frugivoro \\
\hline & Cuniculidae & Cuniculus taczanowskii & Cámara trampa & Frugivoro \\
\hline LAGOMORPHA & Leporidae & Sylvilagus fulvescens & Cámara trampa & Folivoro \\
\hline \multirow[t]{6}{*}{ CHIROPTERA } & \multirow[t]{5}{*}{ Phyllostomidae } & Anoura geoffroyi* & Red de niebla & Nectarivoro \\
\hline & & Carollia brevicauda* & Red de niebla & Frugivoro \\
\hline & & Sturnira cf. ludovici* & Red de niebla & Frugivoro \\
\hline & & Sturnira cf. erythromos* & Red de niebla & Frugivoro \\
\hline & & Artibeus lituratus* & Observación directa & Frugivoro \\
\hline & Vespertilionidae & Myotis cf. oxyotus* & Red de niebla & Insectivoro \\
\hline
\end{tabular}

* Presenta espécimen de referencia depositado en colección de la UPTC.

** Determinación con base en las especies probables, pero esfuerzos adicionales son necesarios para confirmar. 
Los inventarios de mamíferos en la Cordillera Oriental han sido realizados en áreas con distinto grado de intervención y fragmentación de las coberturas vegetales nativas (Zuñiga et al. 1988; Liévano-Latorre \& López-Arévalo 2015; Vargas-Ramírez et al. 2018). Sin embargo, este es el primer estudio que presenta la diversidad de mamiferos en un bosque de roble después de procesos de restauración asistida.

A pesar del limitado esfuerzo de muestreo logramos registrar un número relativamente alto de especies nativas de los Andes (Tabla 1). Los pocos estudios sobre mamíferos en los bosques de roble de la Cordillera Oriental provienen principalmente del análisis de información secundaria, y solo algunas evaluaciones directas en campo (Otálora-Ardila \& López-Arévalo 2006; Vargas-Ramírez et al. 2018). En Santa Bárbara, Santander (2.340 msnm) se encontraron siete especies de pequeños mamíferos no voladores dentro de tres órdenes (Vargas-Ramírez et al. 2018). En un rango altitudinal más amplio (1.600-2.300 msnm), en El Encino, Santander, se encontraron 24 especies de murciélagos (Otálora-Ardila \& López-Arévalo 2006). Otros estudios registraron 24 especies de mamíferos a una menor altitud (1.700-1.800 msnm) en un fragmento de bosque de roble entre las cordilleras Central y Occidental (Ramírez-Chaves \& Pérez 2007) (Tabla 2).

La riqueza de mamíferos en Rogitama refleja cifras similares a otros estudios en bosques andinos de la Cordillera Oriental con altitudes cercanas y tiende a ser mayor que en sitios de menor extensión de bosque. Por ejemplo, en Tenjo y San Francisco, Cundinamarca (2.600 msnm), se registraron dos especies de roedores y un marsupial (Zuñiga et al. 1988). En Tabio, Cundinamarca, (2.650 msnm), se registraron 17 especies entre pequeños y medianos mamíferos no voladores, de los cuales una especie es introducida, Rattus norvegicus (Liévano-Latorre \& López-Arévalo 2015). En Fusagasugá, a una elevación inferior a la del presente estudio, una extensión menor y un esfuerzo de muestreo mayor, se registró una riqueza de pequeños mamíferos similar a la de Rogitama (Ramírez-Chaves et al. 2009) (Tabla 2).

TABLA 2. Número de órdenes y especies de mamíferos de la Reserva Rogitama Biodiversidad, en otros bosques de roble, Quercus humboldtii y en otros tipos de bosque andino en la Cordillera Oriental en Colombia. Se presenta el área de cada sitio, el rango altitudinal y el esfuerzo de muestreo. $\mathrm{pmnv}=$ pequeños mamíferos no voladores; $\mathrm{mm}=$ mamíferos medianos; $\mathrm{h} \cdot \mathrm{m} 2 \cdot \mathrm{r}=$ hora por metro cuadrado red; $t \cdot n=$ trampas por noche; $c \cdot n=$ cámaras por noche.

\begin{tabular}{|c|c|c|c|c|c|c|}
\hline \multicolumn{7}{|c|}{ ESTUDIOS DE MAMIFEROS EN BOSQUES DE ROBLE } \\
\hline Estudio & Localidad & $\begin{array}{l}\text { Área } \\
\text { (ha) }\end{array}$ & $\begin{array}{l}\text { Altitud } \\
(\mathrm{msnm})\end{array}$ & Esfuerzo de muestreo & Órdenes & Especies \\
\hline $\begin{array}{l}\text { Este estudio (Bosque } \\
\text { mixto con dominancia } \\
\text { de roble) }\end{array}$ & $\begin{array}{l}\text { Rogitama } \\
\text { Biodiversidad, } \\
\text { Arcabuco, Boyacá }\end{array}$ & 29 & $2.485-2.550$ & $\begin{array}{l}\text { murciélagos: } 864 \mathrm{~h} \cdot \mathrm{m}^{2} \cdot \mathrm{r} \\
\text { pmnv: } 90 \mathrm{t} \cdot \mathrm{n} \\
\mathrm{mm}: 6 \mathrm{t} \cdot \mathrm{n} ; 6 \mathrm{c} \cdot \mathrm{n}\end{array}$ & 8 & 22 \\
\hline $\begin{array}{l}\text { Otálora-Ardila \& } \\
\text { López-Arévalo, } 2006\end{array}$ & Encino, Santander & 7,06 & $\begin{array}{l}1.600- \\
2.300\end{array}$ & murciélagos: 16 redes & 1 & 24 \\
\hline $\begin{array}{l}\text { Vargas-Ramírez et al., } \\
2018\end{array}$ & $\begin{array}{l}\text { Santa Bárbara, } \\
\text { Santander }\end{array}$ & 1,8 & $2.340^{*}$ & pmnv: $4.312 \mathrm{t} \cdot \mathrm{n}$ & 3 & 7 \\
\hline $\begin{array}{l}\text { Ramírez-Chaves \& } \\
\text { Pérez, } 2007\end{array}$ & $\begin{array}{l}\text { Vereda La Viuda, } \\
\text { Cajibío, Cauca }\end{array}$ & 4,8 & $1.700-1.800$ & $\begin{array}{l}\text { murciélagos: } 57.600 \\
\mathrm{~h} \cdot \mathrm{m} 2 \cdot \mathrm{r} \\
\mathrm{pmnv}: 2.484 \mathrm{t} \cdot \mathrm{n} \\
\mathrm{mm}: 2 \mathrm{t} \cdot \mathrm{n}\end{array}$ & 4 & 24 \\
\hline \multicolumn{7}{|c|}{ OTROS ESTUDIOS EN BOSQUES ANDINOS EN LA CORDILLERA ORIENTAL } \\
\hline $\begin{array}{l}\text { Zuñiga, Rodríguez, \& } \\
\text { Cadena, } 1988\end{array}$ & $\begin{array}{l}\text { Tenjo y San } \\
\text { Francisco, } \\
\text { Cundinamarca }\end{array}$ & 0,25 & 2.600 & $5.904 \mathrm{t} \cdot \mathrm{n}^{*}$ & 2 & 3 \\
\hline $\begin{array}{l}\text { Ramírez-Chaves et al., } \\
2009\end{array}$ & $\begin{array}{l}\text { La Aguadita, } \\
\text { Fusagasugá, } \\
\text { Cundinamarca }\end{array}$ & 15 & $\begin{array}{l}2.300- \\
4.000\end{array}$ & $\begin{array}{l}\text { pmnv: } 1.280 \mathrm{t} \cdot \mathrm{n} \\
\text { murciélagos: } 15.840 \\
\mathrm{hm}^{2} \cdot r\end{array}$ & 2 & 9 \\
\hline
\end{tabular}

Mammalogy Notes 2021, 7(2), 258; https:// doi.org/10.47603/mano.v7n2.258 


\begin{tabular}{|c|c|c|c|c|c|c|}
\hline $\begin{array}{l}\text { Liévano-Latorre \& } \\
\text { López-Arévalo, } 2015\end{array}$ & $\begin{array}{l}\text { Reserva Passiflora, } \\
\text { Tabio, } \\
\text { Cundinamarca }\end{array}$ & 16 & $2.650-2.950$ & $\begin{array}{l}\text { pmnv: } 1.220 \mathrm{t} \cdot \mathrm{n} \\
\mathrm{mm}: 620 \mathrm{c} \cdot \mathrm{n}\end{array}$ & 7 & 17 \\
\hline
\end{tabular}

*Valores estimados con base en la información contenida en la publicación.

Los bosques de roble son ambientes estructurados con múltiples estratos que ofrecen diversidad de recursos alimentarios y de refugios, como epífitas, una capa de hojarasca y cuevas en raíces de árboles y troncos (Nixon 2006). Por ello, para un bosque de roble maduro se esperaría una comunidad de mamíferos con diferentes funciones ecosistémicas, como la reportada en Rogitama, la cual muestra una estructuración taxonómica y funcional. Por ejemplo, especies del género Didelphis, son tolerantes a ambientes transformados y ayudan a conservar y restaurar funciones ecológicas (Cantor et al. 2010; Barrera-Niño \& Sánchez 2014; Barros \& de Aguiar-Azevedo 2014). Mesodepredadores como el tigrillo (Leopardus tigrinus), la comadreja (Neogale frenata) y los zorros (Urocyon cinereoargenteus o Cerdocyon thous), controlan poblaciones de pequeños vertebrados (Eisenberg \& Redford 1989). Especies fosoriales como las musarañas (Cryptotis sp.) y los armadillos (Dasypus novemcinctus) colaboran en las dinámicas del suelo al excavar en búsqueda de invertebrados (de Pascual \& De Ascencao 2000; Rodríguez-Duran et al. 2018). Roedores como el tinajo (Cuniculus taczanowskii), la boruga (Dasyprocta punctata) y la ardilla (Syntheosciurus granatensis) pueden actuar como depredadores y dispersores de semillas en lugares distantes a los de la planta parental (Brewer 2001) y en diferentes estratos como sucede con especies de Rhipidomys (Montenegro-Díaz et al. 1991; Vargas-Ramírez et al. 2018). Además, estos roedores son presa de mamíferos y aves rapaces. Murciélagos frugivoros (Carollia, Sturnira y Artibeus) y nectarivoros (Anoura) desempeñan un papel importante en la composición vegetal al dispersar semillas y polinizar en áreas intervenidas (Gorchov et al. 1993; Galindo-González et al. 2000). Esta diversidad de mamíferos es un indicativo del potencial de las reservas privadas dedicadas a la rehabilitación vegetal en la oferta de recursos naturales y la protección de especies amenazadas.

La restauración de ambientes tropicales de montaña presenta un enorme desafío debido a la alta erosión asociada a las pendientes pronunciadas (Guariguata 2005). La degradación sufrida debido a la erosión y los procesos productivos realizados antes del establecimiento de la Reserva probablemente eliminó una gran parte de los mamíferos silvestres y por ello se habría podido esperar una baja riqueza de mamíferos, ya que estas especies, propias de los bosques andinos colombianos entre 2.400 y $2.600 \mathrm{msnm}$, dependen fuertemente del bosque (López-Arévalo \& Montenegro-Díaz 1993; Sánchez et al. 2004). Sin embargo, después de aproximadamente 40 años de rehabilitación, una muestra de una comunidad de mamíferos típica de los Andes ha recolonizado Rogitama Biodiversidad y ha permitido la rehabilitación de funciones ecosistémicas propias de estos bosques. Investigaciones adicionales deben permitir completar el inventario de mamíferos, evaluar la dinámica de sus poblaciones, determinar su papel en el funcionamiento de los bosques de roble y continuar el seguimiento del proceso de rehabilitación.

\section{AGRADECIMIENTOS}

Los autores agradecen al profesor Gottlied Dandliker por las fotos tomadas en la Reserva. A la profesora Liliana Rosero, a la UPTC y a los coordinadores y asistentes a los cursos sobre mamíferos andinos realizados en 2010 y 2015. Este estudio colabora en la ampliación de la base del conocimiento de la biodiversidad en el país bajo el reto "Uso sostenible de 
la biodiversidad, desarrollo económico y competitividad" dentro del foco Bioeconomía desarrollado en el marco del programa Colombia Científica, gracias al apoyo de la beca Fulbright en los estudios de posgrado de W. Medina. Gracias también a los evaluadores anónimos que hicieron valiosos comentarios para mejorar este documento.

\section{REFERENCIAS}

Armenteras D, Cabrera E, Rodríguez N, Retana J. 2013. National and regional determinants of tropical deforestation in Colombia. Regional Environmental Change. 13(6):1181-1193. https:// doi.org/10.1007/s10113-013-0433-7

Avella-Muñoz A, Cárdenas Camacho LM. 2010. Conservación y uso sostenible de los bosques de roble en el corredor de conservación Guantiva-La Rusia-Iguaque, departamentos de Santander y Boyacá, Colombia. Revista Colombia Forestal. 13(1):5-30.

Barrera-Niño V, Sánchez F. 2014. Forrajeo de Didelphis pernigra (Mammalia: Didelphidae) en un área suburbana de la Sabana de Bogotá, Colombia. Therya. 5(1):289-302. https:// doi.org/10.12933/therya-14-172

Barros FB, de Aguiar-Azevedo P. 2014. Common opossum (Didelphis marsupialis Linnaeus, 1758): Food and medicine for people in the Amazon. Journal of Ethnobiology and Ethnomedicine. 10(1):65. https:// doi.org/10.1186/1746-4269-10-65

Brewer SW. 2001. Predation and dispersal of large and small seeds of a tropical palm. Oikos. 92(2):245-255. https:// doi.org/10.1034/j.1600-0706.2001.920206.x

Cantor M, Ferreira LA, Silva WR, Setz EZF. 2010. Potential seed dispersal by Didelphis albiventris (Marsupialia, Didelphidae) in highly disturbed environment. Biota Neotropica. 10(2):45-51. https:// doi.org/10.1590/S1676-06032010000200004

Clewel AF, Aronson J. 2013. Ecological restoration: principles, values, and structure of an emerging profession. Écoscience. 15(4): 555. https:// doi.org/10.2980/1195-6860-15.4.555.a

de Pascual D, De Ascencao A. 2000. Diet of the cloud forest shrew Cryptotis meridensis (Insectivora: Soricidae) in the Venezuelan Andes. Acta Theriologica. 45(1):13-24.

Devia C, Arenas H. 2000. Evaluación del estatus ecosistémico y de manejo de los bosques de fagáceas (Quercus humboldtii y Trigonobalanus excelsa) en el norte de la Cordillera Oriental (Cundinamarca, Santander y Boyacá). In: Cárdenas F, editor. Cárdenas, TF. Desarrollo sostenible en los Andes de Colombia: Provincias del Norte, Gutiérrez y Valderrama, Boyacá, Colombia. Bogotá, D.C: Instituto de Estudios Ambientales para el Desarrollo, IDEADE, Facultad de Estudios Ambientales y Rurales, Pontificia Universidad Javeriana. p. 301.

Dewi S, Van Noordwijk M, Ekadinata A, Pfund J-L. 2013. Protected areas within multifunctional landscapes: Squeezing out intermediate land use intensities in the tropics? Land Use Policy. 30(1):38-56. https://doi.org/10.1016/j.landusepol.2012.02.006

Díaz-Pulido A, Aguilar-Garavito M, Pérez-Torres J, Solari S. 2015. El monitoreo de los mamíferos en los procesos de restauración ecológica. En: Aguilar-Garavito M. y W. Ramírez (eds.). Monitoreo a procesos de restauración ecológica, aplicado a ecosistemas terrestres. Instituto de Investigación de Recursos Biológicos Alexander von Humboldt (IAvH). Bogotá D.C., Colombia. 250 pp.

Dobson AP, Bradshaw AD, Baker AJM. 1997. Hopes for the future: restoration ecology and conservation biology. Science. 277:515-522. https:// doi.org/10.1126/science.277.5325.515

Eisenberg, J. F., \& Redford, K. H. 1989. Mammals of the Neotropics: The Central Neotropics. Ecuador, Peru, Bolivia, Brazil. University of Chicago Press. Chicago, Ill, USA 
Galindo-González J, Guevara S, Sosa VJ. 2000. Bat-and bird-generated seed rains at isolated trees in pastures in a tropical rainforest. Conservation biology. 14(6):1693-1703. https:// doi.org/10.1111/j.1523-1739.2000.99072.x

Instituto de Hidrología, Meterorología y Estudios Ambientales - IDEAM. "Consulta y Descarga de Datos Hidrometeorológicos - 2021. http:// dhime.ideam.gov.co/atencionciudadano/

Gorchov DL, Cornejo F, Ascorra C, Jaramillo M. 1993. The role of seed dispersal in the natural regeneration of rain forest after strip-cutting in the Peruvian Amazon. Vegetatio. 107(1):339349. https:// doi.org/10.1007/BF00052233

Guariguata M.R. (2005) Restoring Tropical Montane Forests. In: Mansourian S, Vallauri D, Dudley N, eds. Forest Restoration in Landscapes: beyond planting trees. pp. 298-305. Springer, New York, NY. USA. https:// doi.org/10.1007/0-387-29112-1_43

Ibáñez A, Munoz-Mora J, Gafaro M. 2012. Atlas de la distribución de la propiedad rural en Colombia. Universidad de los Andes. Instituto Geográfico Agustín Codazzi (IGAC). Bogotá. Imprenta Nacional de Colombia. $540 \mathrm{p}$.

Liévano-Latorre LF, López-Arévalo HF. 2015. Comunidad de mamíferos no voladores en un área periurbana andina, Cundinamarca, Colombia. Acta Biológica Colombiana. 20(2): 193-202. ttps:// doi.org/10.15446/abc.v20n2.43477

López-Arévalo HF, Montenegro-Díaz OL. 1993. Mamíferos no voladores de carpanta. In: Andrade Gl, editor. Carpanta: selva nublada y páramo. Fundación Natura. Bogotá, Colombia. p. 165-187.

Ministerio de Ambiente y Desarrollo Sostenible. 2015. Plan nacional de restauración: Restauración ecológica, rehabilitación y recuperación de áreas disturbadas. Bogotá, D.C.: Ministerio de Ambiente y Desarrollo Sostenible.

Montenegro-Díaz O, López-Arévalo H, Cadena A. 1991. Aspectos ecológicos del roedor arborícola Rhipidomys latimanus tomes, 1860, (Rodentia: Cricetidae) en el oriente de Cundinamarca, Colombia. Caldasia. 16(79): 565-572.

Narváez G, Sánchez F. 2013. Using foraging behavior to detect edge effects: a case study with Colombian Andean mammals. Boletín Científico del Centro de Museos - Universidad de Caldas. 17(2):110-120.

Nixon KC. 2006. Global and Neotropical distribution and diversity of dak (genus Quercus) and dak forests. In: Kappelle M. (eds). Ecology and conservation of Neotropical montane oak forests. Ecological Studies (Analysis and Synthesis), vol 185. Springer, Berlin, Heidelberg. https:// doi.org/10.1007/3-540-28909-7_1

Otálora-Ardila A. 2003. Mamíferos de los bosques de roble. Acta Biológica Colombiana; 8(2):57-71.

Otálora-Ardila A, López-Arévalo HF. 2006. Incidencia de algunos elementos del paisaje fragmentado de Encino (Santander, Colombia) sobre la riqueza y diversidad de murciélagos. Paper presented at: I Simposio Internacional de Roble y Ecosistemas Asociados, Memorias.

Payán E, de Oliveira T. 2016. Leopardus tigrinus. The IUCN Red List of Threatened Species 2016: eT54012637A50653881.

2.RLTS.T54012637A50653881.en. Accessed on 06 May 2021.

Ramírez-Chaves H, Muñoz-Saba Y, Mendoza-Cifuentes H. 2009. Los mamíferos como herramienta para fundamentar la formulación de planes de manejo y conservación en áreas de jurisdicción de la Corporación Autónoma Regional (CAR). Universidad Nacional de Colombia sede Bogotá y Corporación Autónoma Regional (CAR). Bogotá, Colombia. 50 pp. 
Ramírez-Chaves HE, Pérez WA. 2007. Mamíferos de un fragmento de bosque de roble en el departamento del Cauca, Colombia. Boletín Científico Centro de Museos Museo de Historia Natural; 11(1), 65-79.

Ramírez-Chaves H E, Suárez Castro A F, Morales-Martínez D M, Rodríguez-Posada M E, Zurc D, Concha Osbahr D C, Trujillo A, Noguera Urbano E A, Pantoja Peña G E, González Maya J F, Pérez Torres J, Mantilla Meluk H, López Castañeda C, Velásquez Valencia A, Zárrate Charry D. 2021: Mamíferos de Colombia. v1.12. Sociedad Colombiana de Mastozoología. Dataset/Checklist. https:// doi.org/10.15472/kl1whs

Rodríguez-Durán A, Valencia K, Superina M, Peña R. 2018. Distribución Y usos de los armadillos en sabanas inundables de Arauca, Colombia. Biota Colombiana 19 (2):117-27. https:// doi.org/10.21068/c2018.v19n02a10

Sánchez F, Sánchez-Palomino P, Cadena A. 2004. Inventario de mamíferos en un bosque de los Andes centrales de Colombia. Caldasia. 26:291-309.

Sánchez F, Sánchez-Palomino P, Cadena A. 2008. Species richness and indices of abundance of medium-sized mammals in an Andean forest and reforestations with Andean alder: a preliminary analysis. Caldasia. 30:197-2008.

Sánchez-Cuervo AM, Aide TM. 2013. Identifying hotspots of deforestation and reforestation in Colombia (2001-2010): Implications for protected areas. Ecosphere. 4(11):143. http://dx.doi.org/10.1890/ES13-00207.1

Sikes RS, the Animal Care and Use Committee of the American Society of Mammalogists. 2016 guidelines of the American Society of Mammalogists for the use of wild mammals in research and education. Journal of Mammalogy. 97(3):663-688. https://doi.org/10.1093/jmammal/gyw078

Seabrook L, Mcalpine CA, Bowen ME. 2011. Restore, repair or reinvent: options for sustainable landscapes in a changing climate. Landscape and Urban Planning. 100(4):407-410. https:/ / doi.org/10.1016/j.landurb plan.2011.02.015

Solari S, Muñoz-Saba Y, Rodríguez-Mahecha JV, Defler TR, Ramírez-Chaves HE, Trujillo F. 2013. Riqueza, endemismo y conservación de los mamíferos de Colombia. Mastozoología Neotropical. 20(2):301-365.

Vargas-Ramírez LC, Colmenares-Pinzón JE, Serrano-Cardozo VH. 2018. Uso de microhábitat por un ensamble de pequeños mamíferos no voladores en un bosque de roble sobre la cordillera oriental de los Andes Colombianos. Revista Mexicana de Biodiversidad. 89(2):479-487. https:// doi.org/10.22201/ib.20078706e.2018.2.1933

Zúñiga, H., Rodríguez, J. R., \& Cadena, A. (1988). Densidad de población de pequeños mamíferos en dos comunidades del bosque Andino. Acta Biológica Colombiana, 1(4), 85-93.

\section{Archivos suplementarios}

Editor: Darwin Morales-Martínez Recibido: 2021-06-09

Revisado: 2021-07-01 Aceptado: 2021-09-07 Publicado: 2021-10-18 